\title{
HomSSPICE: A HOMOTOPY-BASED CIRCUIT SIMULATOR FOR PERIODIC STEADY-STATE ANALYSIS OF OSCILLATORS
}

\author{
Wanliang Ma \\ Hewlett Packard \\ 16399 W. Bernardo Dr. \\ San Diego, CA 92127 \\ wanliang_ma@yahoo.com
}

\author{
Ljiljana Trajković \\ School of Engineering Science \\ Simon Fraser University \\ Burnaby, BC V5A 1S6 \\ ljilja@cs.sfu.ca
}

\author{
Kartikeya Mayaram \\ Department of ECE \\ Oregon State University \\ Corvallis, OR 97330 \\ karti@ece.orst.edu
}

\begin{abstract}
In this paper, we describe a new circuit simulator named HomSSPICE that incorporates homotopy algorithms for steady-state simulations of oscillators. The new simulator is based on SSPICE and HOMPACK. It employs a homotopy-based algorithm that is suitable for the periodic steady-state simulations of autonomous circuits, such as sinusoidal oscillators.
\end{abstract}

\section{INTRODUCTION}

With the rapid growth of the radio frequency (RF) integrated circuit market, there is an increasing demand to accurately simulate system performance before fabrication [6,7]. Many system characteristics, such as distortion, power, frequency, and noise, can be properly determined only if the circuit operates in its periodic steady-state domain. For this reason, periodic steady-state simulations of RF circuits are extremely important.

The SPICE simulator can be used to find the steady-state response of circuits only by performing transient analysis over a long period of time, and the designer needs to ensure that the circuit settles into its periodic steady-state. This approach is often inefficient and, in some cases, not suitable for simulating RF circuits. Hence, the periodic steady-state circuit response is simulated directly using either the time-domain NewtonRaphson shooting method, or the frequency-domain harmonic-balance method [7]. The time-domain NewtonRaphson shooting method is preferred for simulating highly nonlinear circuits [2, 3, 6]. However, an appropriate set of initial conditions is required for its convergence. This is a challenging problem when simulating oscillators, where the period of oscillations is not known.

Homotopy methods are robust and accurate numerical techniques for solving nonlinear algebraic equations. They have been used extensively for finding dc operating points of nonlinear circuits [8]. The work of Trajković and Fung $[4,9]$ shows the promise and the first implementation of homotopy methods for periodic steadystate analysis of non-autonomous circuits.

In this paper, we describe a formulation for simulation of autonomous circuits using homotopy algorithms. These algorithms have been implemented in a new simulator called HomSSPICE, derived from SSPICE [3] and HOMPACK [10] (a popular numerical package that implements homotopy algorithms). HomSSPICE is a tool for exploring the use of globally convergent homotopy algorithms for the periodic steady-state analysis of both autonomous and non-autonomous circuits. The simulator is shown to have robust convergence for a variety of sinusoidal oscillators.

\section{HOMOTOPY ALGORITHMS}

Homotopy methods have been widely employed in solving various engineering problems $[1,8,10]$. Suppose that we seek a solution to a system of $n$ nonlinear equations with $n$ variables

$$
F(x)=0
$$

where $F: R^{n} \rightarrow R^{n}$ is a smooth mapping. Let us define a homotopy mapping $H: R^{n} \times R \rightarrow R^{n}$ as

$$
H(x, a, \lambda)=\lambda F(x)+(1-\lambda) g(x-a)
$$

where $G(x):=g(x-a)$ is a smooth mapping whose zeros are easy to find, and $g$ is a constant. Hence,

$$
\begin{aligned}
& H(x, a, 0)=G(x) \\
& H(x, a, 1)=F(x)
\end{aligned}
$$

For $\lambda=1, H(x, a, 1)=F(x)$ is the original problem that needs to be solved. HomSSPICE employs this formulation of the homotopy mapping.

\section{STEADY-STATE ANALYSIS OF OSCILLATORS USING HOMOTOPY METHODS}

Consider the autonomous system

$$
\dot{x}(t)=f(x(t))
$$

where $x$ and $f$ are $n$-dimensional vectors, and $f$ is continuous in $x$ and has continuous first partial derivatives with respect to $x$. Assume that Equation (4) has a non- 
constant periodic solution $w(t)$ of period $T$. Then, for any constant $c, w(t+c)$ is also a solution of Equation (4). Since the period $T$ of the solution is not known, the problem of finding the steady-state solution of an oscillator can be posed as a boundary-value problem

$$
x(0)=x(T)
$$

where period $T$ is a variable.

The solution to Equation (5) can be expressed via the state transition function as $x(t)=\Phi(x(0), 0, t)$, where $x(0)$ is the initial condition. Any solution satisfying Equation (5) will also satisfy

$$
\Phi(x(0), 0, T)=x(0) .
$$

Equation (6) has $n$ equations with $n+1$ unknowns because $T$ is also an unknown.

Aprille and Trick [2] fist proposed an approach to eliminate the additional unknown when applying the Newton-Raphson shooting method to analyze oscillators. This method was subsequently implemented in SSPICE [3]. After a cycle of transient analysis, $n-1$ state variables are selected from the set of $n$ state variables. These $n-1$ unknowns and the unknown period $T$ form the $n$ unknowns for the next step in transient analysis.

However, this approach is not suitable for employing homotopy algorithms because they require fixed initial values. When $\lambda<1$, the transient solutions obtained by using the homotopy approach are not the actual transients of the original system of equations. Furthermore, although only $n$ unknowns are used in the shooting method, these unknowns are not fixed. The $n$ unknowns used for a cycle of transient analysis may change at the beginning of the next transient analysis cycle. With the homotopy approach, the $n$ unknowns must be determined in advance, and they must remain unchanged during the entire solution process. Therefore, eliminating one unknown when applying the NewtonRaphson shooting method is not applicable when using homotopy algorithms.

Kundert et al., [5] proposed adding an equation when applying the Newton-Raphson shooting method. The period $T$ is added to the list of unknowns. An extra equation is added to remedy the problem of obtaining a continuum of solutions. This extra equation restricts the set of solutions so that $x(0)$ lies on a hyperplane. The set of equations to be solved becomes

$$
\left\{\begin{array}{c}
\Phi(x(0), 0, T)=x(0) \\
q^{T} x(0)=a
\end{array}\right.
$$

where $q$ is a constant vector normal to the hyperplane, and $a$ is a scalar. The added equation needs to be carefully selected so that the hyperplane intersects the solution trajectory. Therefore, $q$ and $a$ cannot be chosen arbitrarily, and should satisfy the following conditions:

$$
\left\{\begin{array}{c}
\max _{t} q^{T} x(t)>a \\
\min _{t} q^{T} x(t)<a . \\
q^{T} \frac{d x(0)}{d t} \neq 0
\end{array}\right.
$$

This approach is suitable for employing homotopy algorithms because the added equation limits the range of orbits of the state variables in an autonomous circuit. The added equation restricts the remaining $n$ equations, and the search for the solutions is limited to a range of the solution orbits, rather than to the complete solution space. Unfortunately, the range of the solution orbits is not known a priori. The authors [5] suggest that this range should be searched gradually when running transient analyses by updating the vector $a$ as the iterations progress. This requirement makes the approach unsuitable for applying homotopy algorithms.

\section{HOMOTOPY FORMULATION FOR STEADY- STATE ANALYSIS OF OSCILLATORS}

A modification of Equations (7) and (8) enables the use of the homotopy algorithms with the Newton-Raphson shooting method.

The key idea is to use $q$ and $a$ known in advance, rather than to search their values gradually as suggested in [5]. Suppose that $q_{i}=1$ and $q_{j \neq i}=0$. The second Equation (7) then becomes

$$
x_{i}(0)=a .
$$

Hence, this modification reduces the problem to finding the orbit of only one state variable, rather than finding the orbits of all state variables.

The value of $a$ also needs to be determined. Suppose that the solution of $x_{i}$ is a sinusoid, as shown in Figure 2. The constraint Equation (8) becomes

$$
\min \left(x_{i}\right)<a<\max \left(x_{i}\right) .
$$

Hence, $x_{i}(0)$ cannot assume the values of the local extreme points, such as points $\mathrm{A}, \mathrm{B}$, and $\mathrm{C}$ in Figure 1. In practice, the value of $a$ is obtained from a transient analysis of the circuit. An experienced designer may also be able to specify its value.

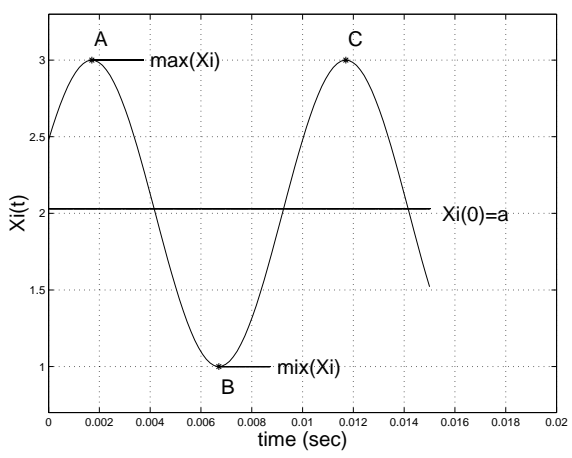

Figure 1: Solution for the state variable $x_{i}$. 


\section{IMPLEMENTATION AND RESULTS}

The new homotopy-based formulation described in Section 4 has been implemented in a new simulator called HomSSPICE, derived from SSPICE [3] and HOMPACK [10]. In this implementation, Equation (2) was used as the homotopy mapping, and Equations (6) and (9) were used to define the original problem to be solved. HOMPACK is a suite of homotopy algorithms and the problem to be solved may be formulated as a fixed-point, zero-finding, or curve-tracking problem. Furthermore, HOMPACK supports three algorithms: FIXPDF (based on ordinary differential equation), FIXPQF (based on augmented Jacobian), and FIXPNF (based on the normal flow algorithm).

The HomSSPICE periodic steady-state solutions for the phase-shift oscillator shown in Figure 2 are given in Table 1. The period calculated by the Newton-Raphson based shooting method is $\mathrm{T}=4.647858 \mathrm{e}-3$ seconds, with an execution time of 1.08 seconds on a HP 712/60 workstation.

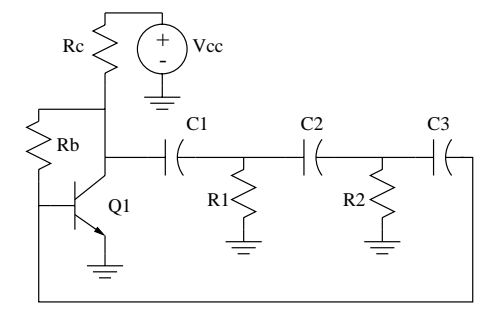

Figure 2: Phase-shift oscillator. $\mathrm{Rc}=3.3 \mathrm{~K} \Omega, \mathrm{Rb}=470 \mathrm{~K} \Omega, \mathrm{R} 1$ $=7 \mathrm{~K} \Omega, \mathrm{R} 2=5 \mathrm{~K} \Omega, \mathrm{C} 1=\mathrm{C} 2=\mathrm{C} 3=47 \mathrm{nF}$, and $\mathrm{Vcc}=10 \mathrm{~V}$.

As shown in Table 1, the periods computed by using the three homotopy-based algorithms are in good agreement (up to the fifth decimal place). All three approaches produced solutions that satisfy Equation (9). In terms of execution time, the FIXPNF algorithm is the most efficient, followed by the FIXPQF algorithm and the (slowest) FIXPDF algorithm. For this circuit, the homotopy methods required much more computational effort than the Newton-Raphson shooting method. The solution traces for the zero-finding problem with the FIXPDF algorithm are shown in Figure 3.

Simulation results for various oscillator circuits are summarized in Table 2. These are examples of circuits that converged when simulated using the NewtonRaphson shooting method. We used them to estimate the speed performance of our homotopy-based shooting method. However, there are oscillators that could not be simulated using the Newton-Raphson based shooting method because the method fails to converge. The combination of FIXPDF and the curve-tracking option proved to be the best approach for solving these difficult circuits. Using this combination, we were able to find steady-state solutions of oscillators for which the Newton-
Raphson shooting method did not converge. HomSSPICE results for these circuits are summarized in Table 3. These examples demonstrate that the periodic steady-state analysis using homotopy methods can provide solutions for difficult oscillator circuits.

\section{CONCLUSION}

A key contribution of this paper is the development and implementation of homotopy-based periodic steady-state simulations of oscillator circuits. It describes the first successful implementation of homotopy methods for simulation of autonomous circuits. A new formulation based on homotopy algorithms has been developed and implemented in the circuit simulator HomSSPICE, derived from SSPICE and HOMPACK. We have simulated a variety of circuit examples, and demonstrated that homotopy methods offer improved convergence for periodic steady-state simulations of oscillators.

\section{REFERENCES}

[1] E. L. Allgower and K. Georg, Numerical continuation methods: An introduction, New York, NY: Springer-Verlag, 1990.

[2] T. J. Aprille and T. N. Trick, "A computer algorithm to determine the steady-state response of nonlinear oscillators," IEEE Trans. Circuit Theory, vol. CT-19, pp. 354-360, July 1972.

[3] P. N. Ashar, "Implementation of algorithms for the periodic steady-state analysis of nonlinear circuits," ERL Memo $U C B / E R L$ M89/31, The University of California at Berkeley, March 1989.

[4] E. Fung, "Homotopy methods for dc operating point and periodic steady-state analysis: SPICE3 implementation," M.Sc. Thesis, Dept. of EECS, University of California, Berkeley, 1998.

[5] K. S. Kundert, J. K. White, and A. Sangiovanni-Vincentelli, Steady-State Methods for Simulating Analog and Microwave Circuits. Boston, MA: Kluwer Academic Publishers, 1990.

[6] K. S. Kundert, "Introduction to RF simulation and its application," IEEE J. Solid-State Circuits, pp. 1298-1319, Sept. 1999.

[7] K. Mayaram, D. C. Lee, S. Moinian, D. Rich, and J. Roychowdhury, "Computer-aided circuit analysis tools for RFIC simulation: algorithms, features, and limitations," IEEE Trans. Circuits and Systems-II, pp. 274-286, April 2000.

[8] Lj. Trajković, "Homotopy methods for computing dcoperating points," Encyclopedia of Electrical and Electronics Engineering, J. G. Webster, Ed., New York: John Wiley \& Sons, vol. 9, pp. 171-176, 1999.

[9] Lj. Trajković, E. Fung, and S. Sanders, "HomSPICE: simulator with homotopy algorithms for finding $\mathrm{dc}$ and steady-state solutions of nonlinear circuits," Proc. IEEE Int. Symp. Circuits and Systems, June 1998, TPA 10-2.

[10] L. T. Watson, S. C. Billups, and A. P. Morgan, "Algorithm 652: HOMPACK: a suit of codes for globally convergent homotopy algorithms," ACM Trans. Mathematical Software, vol. 13, no. 3, pp. 281-310, Sept. 1987. 


\begin{tabular}{|l|l|l|l|}
\hline & FIXPDF with zero-finding & FIXPNF with curve-tracking & FIXPQF with zero-finding \\
\hline No. of Jacobian eval. & 166 & 45 & 11 \\
\hline Execution Time $(\mathrm{sec})$ & 30.11 & 6.45 & 8.62 \\
\hline Lambda & $1.000000 \mathrm{e}+00$ & $1.000000 \mathrm{e}+00$ & $1.000000 \mathrm{e}+00$ \\
\hline Arc length & 1.191 & 1.182 & 1.190 \\
\hline Calculated T $(\mathrm{sec})$ & $4.647837 \mathrm{e}-03$ & $4.647832 \mathrm{e}-03$ & $4.647832 \mathrm{e}-03$ \\
\hline
\end{tabular}

Table 1: Periodic steady-state simulations of the phase-shift oscillator using homotopy methods implemented in HomSSPICE. CPU times are given for an HP 712/60 workstation.
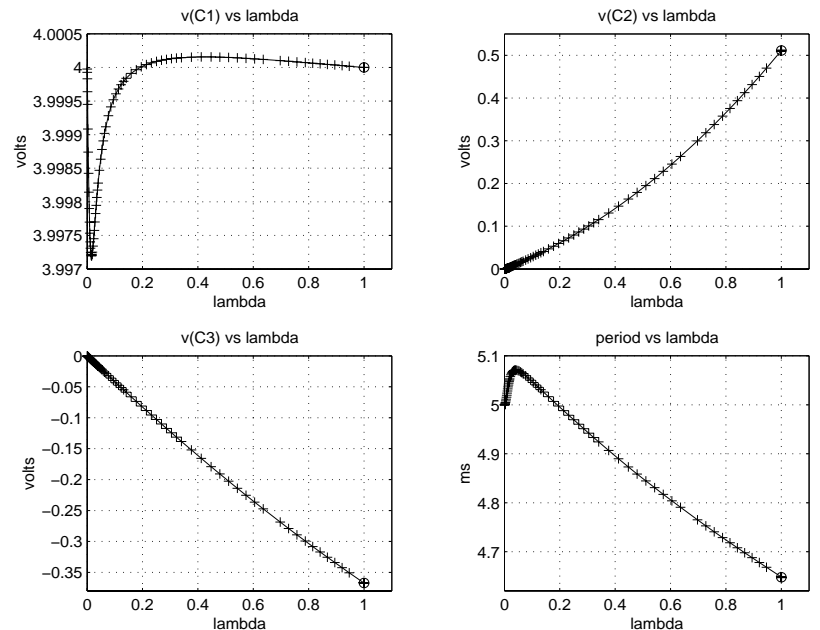

Figure 3: Solution traces for the phase-shift oscillator using HomSSPICE with the zero-finding option and the FIXPDF algorithm.

\begin{tabular}{|l|c|c|c|c|}
\hline \multicolumn{1}{|c|}{ Oscillator circuit } & \multicolumn{2}{c|}{ Calculated period (sec) } & \multicolumn{2}{c|}{ CPU time (sec) } \\
\cline { 2 - 5 } & Newton method & Homotopy method & Newton method & Homotopy method \\
\hline Phase-shift & $4.647858 \mathrm{E}-03$ & $4.647837 \mathrm{E}-03$ & 1.1 & 30.1 \\
\hline High frequency Colpitts & $1.273496 \mathrm{E}-09$ & $1.215747 \mathrm{E}-09$ & 2.6 & 13.1 \\
\hline Colpitts $(\mathrm{Q}=50)$ & $6.251622 \mathrm{E}-07$ & $6.251402 \mathrm{E}-07$ & 9.7 & 24.5 \\
\hline Colpitts $(\mathrm{Q}=100)$ & $6.291605 \mathrm{E}-07$ & $6.291590 \mathrm{E}-07$ & 2.6 & 23.9 \\
\hline 62 MHz & $1.625924 \mathrm{E}-08$ & $1.626752 \mathrm{E}-08$ & 5.1 & 23.8 \\
\hline TNT & $1.128189 \mathrm{E}-04$ & $1.127167 \mathrm{E}-04$ & 3.0 & 37.7 \\
\hline Wein & $6.602231 \mathrm{E}-06$ & $6.604525 \mathrm{E}-06$ & 1.1 & 11.6 \\
\hline Op amp-based Wein & $1.002995 \mathrm{E}-04$ & $1.002840 \mathrm{E}-04$ & 1.2 & 6.9 \\
\hline Emitter-coupled & $2.080204 \mathrm{E}-07$ & $2.093521 \mathrm{E}-07$ & 3.9 & 22.4 \\
\hline MOSFET & $1.643657 \mathrm{E}-08$ & $1.652160 \mathrm{E}-08$ & 1.2 & 33.1 \\
\hline
\end{tabular}

Table 2: Comparison of Newton-Raphson and HomSSPICE homotopy-based shooting methods for finding periodic steady-state solutions of various oscillator circuits. CPU times are given for an HP 712/60 workstation.

\begin{tabular}{|l|c|c|}
\hline \multicolumn{1}{|c|}{ Oscillator circuit } & Calculated period $(\mathrm{sec})$ & CPU time $(\mathrm{sec})$ \\
\hline CMOS negative-Gm & $5.796000 \mathrm{E}-10$ & 950 \\
\hline Colpitts $(\mathrm{Q}=1000)$ & $6.291833 \mathrm{E}-08$ & 25.9 \\
\hline Colpitts $(\mathrm{Q}=1020)$ & $5.167606 \mathrm{E}-04$ & 72.9 \\
\hline Colpitts $(\mathrm{Q}=10000)$ & $1.622373 \mathrm{E}-09$ & 32.9 \\
\hline CMOS SC pair & $1.000893 \mathrm{E}-08$ & 19.2 \\
\hline
\end{tabular}

Table 3: Steady-state solutions of oscillator circuits obtained using HomSSPICE. Newton-Raphson method fails to converge for these circuits. 